\title{
Effect of vancomycin loading dose on clinical outcome in critically ill patients with methicillin-resistant Staphylococcus aureus pneumonia
}

\author{
Jin Gu Yoon ${ }^{1 \#}$, Kyungmin Huh ${ }^{2 \#}$, You Min Sohn ${ }^{3}$, Hyo Jung Park ${ }^{3}$, Soo Jin Na ${ }^{1}$, Kyeongman Jeon ${ }^{1,4} \wedge$ \\ ${ }^{1}$ Department of Critical Care Medicine, Samsung Medical Center, Sungkyunkwan University School of Medicine, Seoul, Republic of Korea; \\ ${ }^{2}$ Division of Infectious Diseases, Department of Medicine, Samsung Medical Center, Sungkyunkwan University School of Medicine, Seoul, Republic \\ of Korea; ${ }^{3}$ Department of Pharmaceutical Services, Samsung Medical Center, Sungkyunkwan University School of Medicine, Seoul, Republic of \\ Korea; ${ }^{4}$ Division of Pulmonary and Critical Care Medicine, Department of Medicine, Samsung Medical Center, Sungkyunkwan University School of \\ Medicine, Seoul, Republic of Korea \\ Contributions: (I) Conception and design: JG Yoon, K Huh, K Jeon; (II) Administrative support: K Jeon; (III) Provision of study materials or patients: \\ JG Yoon, K Huh; (IV) Collection and assembly of data: JG Yoon, K Huh, YM Sohn, HJ Park; (V) Data analysis and interpretation: All authors; (VI) \\ Manuscript writing: All authors; (VII) Final approval of manuscript: All others \\ "These authors contributed equally to this work. \\ Correspondence to: Kyeongman Jeon, MD, PhD. Division of Pulmonary and Critical Care Medicine, Department of Medicine and Critical Care \\ Medicine, Samsung Medical Center, Sungkyunkwan University School of Medicine, 81 Irwon-ro, Gangnam-gu, Seoul 06351, Republic of Korea. \\ Email: kjeon@skku.edu.
}

Background: Vancomycin is the treatment of choice for serious methicillin-resistant Staphylococcus aureus (MRSA) infections. Current guidelines recommend giving an initial loading dose (LD) of $25-30 \mathrm{mg} / \mathrm{kg}$ to rapidly increase the serum concentration. However, high-quality evidence for the clinical benefit of LD is lacking. Herein, we aim to examine the association between vancomycin LD and clinical outcome.

Methods: A retrospective cohort study was conducted on adult patients treated for MRSA pneumonia with vancomycin in medical intensive care units from April 2016 to August 2018. MRSA pneumonia was defined by the Centers for Disease Control and National Healthcare Safety Network definition. The primary outcome was the clinical cure of pneumonia. Secondary outcome measures included time to pharmacokinetic (PK) target attainment, microbiological cure, acute kidney injury, and all-cause mortality.

Results: A total of 81 patients were included; of these $22(27.2 \%)$ received LD. The mean initial dose was significantly higher in the LD group. Clinical cure was similar in both groups $(68.2 \%$ vs. $66.1 \%$ in the LD and non-LD groups, respectively; $\mathrm{P}=0.860$ ). No significant difference was observed in the microbiological cure, all-cause mortality, and incidence of acute kidney injury. Furthermore, no difference was observed in terms of time to PK target attainment (69.2 vs. $63.4 \mathrm{~h}$ in the LD and non-LD groups, respectively; $\mathrm{P}=0.624)$. Vancomycin minimum inhibitory concentration of $<2 \mathrm{mg} / \mathrm{L}$ was identified as an independent predictive factor for clinical cure in multivariable analysis, whereas vancomycin LD was not.

Conclusions: Initial LD is not associated with better clinical outcome or rapid pharmacological target attainment in critically ill patients with MRSA pneumonia. Further studies are warranted to provide better evidence for this widely recommended practice.

Keywords: Vancomycin; pharmacokinetics; methicillin-resistant Staphylococcus aureus; pneumonia; critical illness

Submitted Jun 18, 2020. Accepted for publication Nov 02, 2020.

doi: $10.21037 /$ jtd-20-2243

View this article at: http://dx.doi.org/10.21037/jtd-20-2243

^ ORCID: 0000-0002-4822-1772. 


\section{Introduction}

Methicillin-resistant Staphylococcus aureus (MRSA) is one of the most frequently identified pathogens causing hospitalacquired pneumonia (HAP) and ventilator-associated pneumonia (VAP) worldwide (1-3). In critically ill patients, MRSA pneumonia is associated with significant morbidity and mortality $(4,5)$. Vancomycin is a drug of choice for serious MRSA infections and has been widely used as an empirical and definitive antibiotic in critically ill patients. However, complicated pharmacokinetic/pharmacodynamic (PK/PD) parameter of the drug led to controversies on optimal drug dosage and interval (6). The most accurate $\mathrm{PK} / \mathrm{PD}$ indicator for effective bacterial killing by vancomycin is known to be an area under the curve (AUC) to minimum inhibitory concentration (MIC), as AUC/MIC $\geq 400$ has been shown to be associated with better clinical outcomes $(7,8)$. However, multiple measurements of peak and trough concentration is required to estimate AUC/ MIC, making it less practical in real-world settings. In practice, vancomycin serum trough concentration $\left(\mathrm{C}_{\text {trough }}\right)$ of $15-20 \mathrm{mg} / \mathrm{L}$ has been widely used as a surrogate marker for the ideal AUC/MIC level (9).

The previous guideline on the dosage and monitoring of vancomycin commonly recommend an initial loading dose (LD) of $25-30 \mathrm{mg} / \mathrm{kg}$ actual body weight (ABW) to achieve rapid attainment of target concentration in critically ill patients (9), although the dose has been changed to 20-35 $\mathrm{mg} / \mathrm{kg}$ in the recent guideline (10). Several pharmacological studies have shown that the initial LD of vancomycin is associated with more rapid attainment of target $\mathrm{C}_{\text {trough }}$ in critically ill patients, which in theory may reduce the chance of treatment failure and emergence of resistance (11-13). However, direct evidence showing that vancomycin LD improves clinical outcome, especially in critically ill patients with MRSA pneumonia, are lacking $(14,15)$. In addition, vancomycin LD might cause more nephrotoxicity than conventional dose in patients with higher risk for kidney injury, which is a particularly important concern in intensive care units (ICUs) (16). The pharmacological action of vancomycin would be even more complicated in critically ill patients, as increased volume of distribution and augmented or decreased renal clearance may substantially alter the PK/PD profiles of antibiotics (17). Thus, evidence on the effect of vancomycin LD on clinical outcomes are urgently needed.

Herein, we aim to examine whether the initial LD of vancomycin is associated with improved clinical outcome in critically ill patients with microbiologically confirmed MRSA pneumonia. In addition, the PK target attainment evaluated by $\mathrm{C}_{\text {trough }}$ and incidence of nephrotoxicity between $\mathrm{LD}$ and non-LD groups were investigated. We present the following article in accordance with the STROBE reporting checklist (available at http://dx.doi.org/10.21037/jtd-202243).

\section{Methods}

\section{Study design and population}

This observational study was conducted at Samsung Medical Center (a 1,989-bed, university-affiliated, tertiary referral hospital in Seoul, South Korea) between April 2016 and August 2018. Adult patients diagnosed with HAP or VAP caused by MRSA and treated with intravenous vancomycin in medical ICUs were retrospectively reviewed. Patients were included if all the following conditions were satisfied: (I) admitted to the ICU, (II) MRSA was identified from lower respiratory specimen (sputum, tracheal aspiration, and bronchoalveolar lavage) as a pathogen in microbiological tests, and (III) received intravenous vancomycin for the treatment of pneumonia for at least $72 \mathrm{~h}$.

The study was conducted in accordance with the Declaration of Helsinki (as revised in 2013). The Institutional Review Board at Samsung Medical Center approved the study (IRB No. 2018-05-184) and waived the requirement for informed consent because of the observational nature of the study. All patient records and data were anonymized and de-identified before analysis.

\section{Diagnosis of pneumonia and microbiological tests}

Pneumonia was diagnosed when a new and progressive pulmonary infiltrate on chest radiography was accompanied by clinical evidence, including fever, purulent sputum, leukocytosis, or decrease in oxygenation (18). HAP was defined as pneumonia that developed more than $48 \mathrm{~h}$ after admission. VAP was defined as pneumonia that developed more than $48 \mathrm{~h}$ after endotracheal intubation.

If a patient was diagnosed with pneumonia, respiratory specimens for quantitative culture were obtained before initiation of new antibiotics. Respiratory specimens included sputum, transtracheal aspirate, bronchoalveolar lavage fluid, and pleural fluid. The Clinical and Laboratory Standards Institute interpretive criteria were used to determine antimicrobial susceptibilities. Follow-up cultures were 
performed at least $72 \mathrm{~h}$ after the initiation of vancomycin treatment to assess the microbiological response. The minimum inhibitory concentration (MIC) of MRSA isolates was determined by broth microdilution (VITEK 2, bioMérieux, France).

\section{Treatment regimen}

In the hospital, before the hospital guidelines were established, patients received intravenous vancomycin using a physician-selected vancomycin dosage regimen without an LD. As the guidelines for vancomycin dosing were developed in April 2017, physicians used an LD to attain target trough serum vancomycin levels earlier, based on the individual physician's discretion (9). The hospital's guidelines recommended an LD of $25 \mathrm{mg} / \mathrm{kg}$ followed by $15-20 \mathrm{mg} / \mathrm{kg}$ every $12 \mathrm{~h}$ and then adjusted for the target $\mathrm{C}_{\text {trough }}$ of $15-20 \mathrm{mg} / \mathrm{L}$. LD never exceeded 2,000 mg even if the patient weighed more than $80 \mathrm{~kg}$. For patients with impaired renal function [creatinine clearance $(\mathrm{CrCl})$ $\left.<50 \mathrm{~mL} / \mathrm{min} / 1.73 \mathrm{~m}^{2}\right]$, the maintenance dose was adjusted on the concentration at $12 \mathrm{~h}$ after LD. Vancomycin concentrations were analyzed using a kinetic interaction of microparticles in a solution (cobas c702 module, Roche Diagnostics GmbH, Germany). However, the frequency of $\mathrm{C}_{\text {trough }}$ was left to the clinical pharmacist's discretion during the ICU multidisciplinary team rounding.

\section{Data collection and outcome measures}

Clinical, laboratory, and outcome data were collected by a retrospective review of electronic hospital records. Demographic data, including age, sex, body mass index (BMI), comorbidity, immune state, sequential organ failure assessment score, and antibiotic susceptibility, were recorded on the day of first administration of intravenous vancomycin. Data on the use of concomitant nephrotoxic agents and other antibiotics and the initiation of renal replacement therapy (RRT) during intravenous vancomycin therapy were also collected. Baseline $\mathrm{CrCl}$ was calculated by Cockcroft-Gault and Modification of Diet in Renal Disease equations (19).

The primary outcome was the clinical cure of MRSA pneumonia, which was defined as the improvement of all signs and symptoms associated with pneumonia, including fever, laboratory abnormalities (leukocytosis and elevated C-reactive protein), and pulmonary infiltrates on chest radiographs. The clinical response of all patients was assessed by two authors who were not aware of which treatment was given to the patient. In the event of a discrepancy, two reviewers discussed and reached a consensus. The secondary outcomes included microbiological cure defined as the absence of MRSA in the final culture of specimens during hospitalization, time to PK target attainment calculated as the time between the first dose of vancomycin and the first measurement of $\mathrm{C}_{\text {trough }}>15 \mu \mathrm{g} / \mathrm{mL}$, nephrotoxicity during vancomycin therapy, initiation of RRT, and mortalities. Nephrotoxicity was defined as a risk, injury, failure, loss of kidney function, and end-stage kidney disease classification of injury or more, with injury defined as a greater than twofold increase in serum creatinine, a greater than $50 \%$ reduction in glomerular filtration rate compared to the value at the start of treatment, or oliguria $(\leq 0.5 \mathrm{~mL} / \mathrm{kg} / \mathrm{h})$ for $\geq 12 \mathrm{~h}(20,21)$. Patients on RRT at the time of intravenous vancomycin initiation were excluded from nephrotoxicity analysis.

\section{Statistical analysis}

Data are presented as median with interquartile range (IQR) for continuous variables and number (\%) for categorical variables. Data were compared using $\chi^{2}$ or Fisher's exact test for categorical variables and Student's $t$-test or MannWhitney test for continuous variables, as appropriate. Logistic regression analysis was performed to identify the variables associated with clinical outcomes. Variables with $\mathrm{P}<0.2$ on univariate analyses (22), as well as a priori variables that were clinically relevant, were entered into a multiple logistic regression model in which early target attainment within $48 \mathrm{~h}$, clinical cure, or acute kidney injury was the outcome variable of interest. Odds ratios (ORs) with $95 \%$ confidence intervals (95\% CIs) were calculated. For the subgroup analysis of patients with $\mathrm{CrCl}>30 \mathrm{~mL} / \mathrm{min}$, each case in LD group was matched to a control in non-LD group by a $\mathrm{CrCl}$ with a caliper of $15 \mathrm{~mL} / \mathrm{min}$. All tests were two-tailed and $\mathrm{P}<0.05$ was considered significant. SPSS Statistics version 25 (IBM, Armonk, NY, USA) were used for analysis.

\section{Results}

\section{Baseline clinical characteristics}

Of the 81 patients who were eligible for analysis, 22 (27.2\%) patients received vancomycin $\mathrm{LD}$. The baseline clinical characteristics of LD and non-LD groups are summarized in Table 1. LD group had better renal function at baseline 
Table 1 Baseline characteristics of critically ill patients with or without vancomycin loading dose for MRSA pneumonia

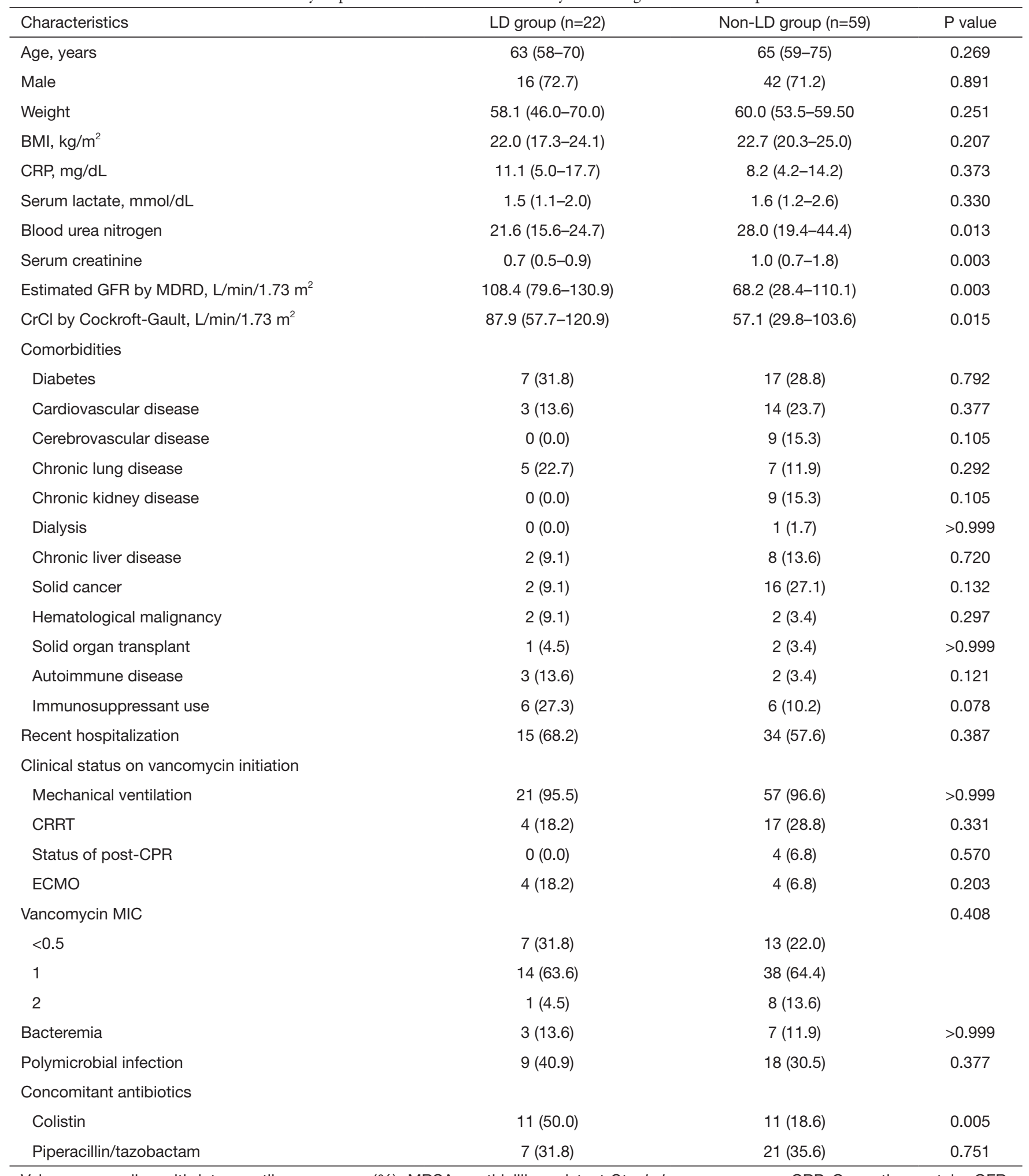

Values are median with interquartile range or $\mathrm{n}(\%)$. MRSA, methicillin-resistant Staphylococcus aureus; CRP, C-reactive protein; GFR, glomerular filtration rate; MDRD, Modification of Diet in Renal Disease; CrCl, creatinine clearance; CRRT, continuous renal replacement therapy; CPR, cardiopulmonary resuscitation; ECMO, extracorporeal membrane oxygenation; MIC, minimum inhibitory concentration. 
Table 2 Vancomycin dose and $\mathrm{C}_{\text {trough }}$ measurement between loading dose (LD) and non-LD groups

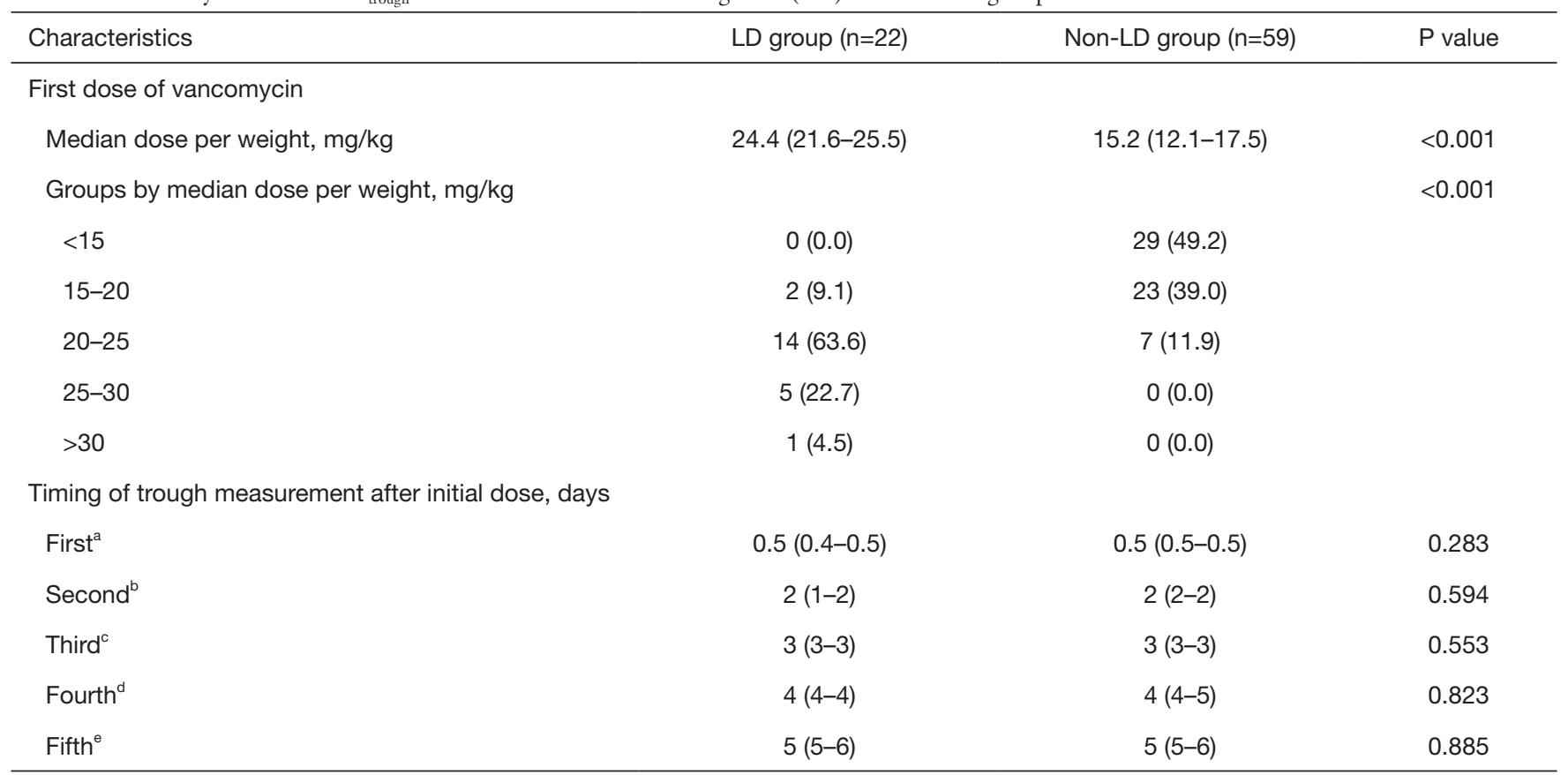

Values are median with interquartile range or $n(\%) .{ }^{a} n=22$ and 54 , respectively; ${ }^{b} n=21$ and 53 , respectively; ${ }^{c} n=21$ and 49 , respectively; ${ }^{d} \mathrm{n}=19$ and 47 , respectively; ${ }^{e} \mathrm{n}=16$ and 44 , respectively.

compared to non-LD group. However, the prevalence of chronic kidney disease or dialysis was not significantly different between the two groups. Needs for organ support, including mechanical ventilation, RRT, and extracorporeal membrane oxygenation, and the severity of pneumonia were similar between the two groups. The distribution of vancomycin MIC of MRSA isolates was also comparable between the two groups. However, colistin was coadministered more frequently in LD group compared to non-LD group ( $50.0 \%$ vs. $18.6 \%, \mathrm{P}=0.005)$.

\section{Vancomycin treatment}

LD group received a significantly higher initial dose of vancomycin [24.4 (21.6-25.5) vs. $15.2(12.1-17.5) \mathrm{mg} / \mathrm{kg}$ $\mathrm{ABW}, \mathrm{P}<0.001$; Table 2]. The timing of subsequent $\mathrm{C}_{\text {trough }}$ measurements did not differ significantly between the two groups. Despite 1.6 times higher mean weight-based dose of the first vancomycin, LD group did not reach PK target earlier compared to non-LD group (Table 3). Both groups showed similar median time to target attainment [54 h (24-110) vs. $60 \mathrm{~h}$ (24-93), $\mathrm{P}=0.981]$ and proportion of patients attaining target within $24 \mathrm{~h}(28.6 \%$ vs. $30.9 \%, \mathrm{P}=0.843)$. In a multiple logistic regression model for variables associated with early target attainment within $48 \mathrm{~h}$ from the initial vancomycin dose, lower $\mathrm{CrCl}$ (adjusted OR 0.98, 95\% CI: $0.97-0.99, \mathrm{P}=0.003)$ was identified as an independent predictive factor (Table 4). However, vancomycin LD was only marginally associated with early target attainment (adjusted OR 2.76, 95\% CI: 0.84-9.07, $\mathrm{P}=0.096$ ).

\section{Clinical outcomes}

Clinical outcomes were also similar between the two groups. The primary outcome of clinical cure was not significantly better in LD group compared to non-LD group $(68.2 \%$ vs. $66.1 \%, \mathrm{P}=0.860)$. Two groups showed comparable microbiological cure rates $(72.7 \%$ vs. $86.4 \%$, $\mathrm{P}=0.188)$. However, 90-day mortality tended to be higher in LD group than in non-LD group (36.4\% vs. $18.6 \%$, $\mathrm{P}=0.094)$, although ICU mortality was not different between the two groups ( $18.2 \%$ vs. $15.3 \%, \mathrm{P}=0.742)$. The results of univariable and multivariable analyses with the multiple logistic regression model for the probability of clinical cure are presented in Table 5. After adjusting for potential confounding factors, vancomycin MIC $<2 \mathrm{mg} / \mathrm{dL}$ was independently associated with clinical cure (adjusted OR 0.19, 95\% CI: 0.04-1.00, $\mathrm{P}=0.050$ ), whereas 
Table 3 Proportion of pharmacokinetic target (trough concentration $>15 \mathrm{mg} / \mathrm{L}$ ) attainment between vancomycin loading dose (LD) and non-LD groups

\begin{tabular}{|c|c|c|c|c|}
\hline Characteristics & LD group $(\mathrm{n}=22)$ & Non-LD group $(n=59)$ & OR $(95 \% \mathrm{Cl})$ & $P$ value \\
\hline Target attainment within $24 \mathrm{~h}$ & $6(28.6)$ & $17(30.9)$ & $0.89(0.30-2.70)$ & 0.843 \\
\hline Target attainment within $48 \mathrm{~h}$ & $10(47.6)$ & $22(40.0)$ & $1.36(0.50-3.75)$ & 0.547 \\
\hline
\end{tabular}

Values are median with interquartile range or $\mathrm{n}(\%)$. OR, odds ratio; $\mathrm{Cl}$, confidence interval

Table 4 Univariable and multivariable analyses with logistic regression model for probability of pharmacokinetic target $(\geq 15 \mathrm{mg} / \mathrm{L})$ attainment within $48 \mathrm{~h}$

\begin{tabular}{|c|c|c|c|c|}
\hline Variables & \multicolumn{2}{|c|}{ Univariable } & \multicolumn{2}{|c|}{ Multivariable } \\
\hline Age, per year & NA & 0.291 & & \\
\hline Sex, female & $0.93(0.34-2.55)$ & 0.893 & & \\
\hline Vancomycin LD & $1.36(0.50-3.75)$ & 0.547 & $2.76(0.84-9.07)$ & 0.096 \\
\hline CRRT & $1.17(0.42-3.29)$ & 0.760 & & \\
\hline ECMO & $1.43(0.33-6.20)$ & 0.714 & & \\
\hline
\end{tabular}

OR, odds ratio; $\mathrm{Cl}$, confidence interval; $\mathrm{LD}$, loading dose; $\mathrm{CrCl}$, creatinine clearance; $\mathrm{CRRT}$, continuous renal replacement therapy; ECMO, extracorporeal membrane oxygenation. *Per each $\mathrm{mL} / \mathrm{min}$ increase.

Table 5 Univariable and multivariable analyses with logistic regression model for probability of clinical cure

\begin{tabular}{|c|c|c|c|c|}
\hline Variables & \multicolumn{2}{|c|}{ Univariable } & \multicolumn{2}{|c|}{ Multivariable } \\
\hline Age, per year & $\mathrm{N} / \mathrm{A}$ & 0.486 & $0.97(0.93-1.01)$ & 0.109 \\
\hline Sex, female & $1.20(0.43-3.40)$ & 0.727 & $1.52(0.47-4.94)$ & 0.484 \\
\hline Vancomycin LD & $1.10(0.39-3.13)$ & 0.860 & $0.91(0.27-2.99)$ & 0.870 \\
\hline Cardiovascular disease & $2.80(0.73-10.76)$ & 0.123 & 4.79 (0.99-23.17) & 0.051 \\
\hline Mechanical ventilation & $1.00(0.87-11.54)$ & $>0.999$ & & \\
\hline CRRT & $0.33(0.12-0.93)$ & 0.031 & $0.31(0.09-1.04)$ & 0.058 \\
\hline Status of post-CPR & $0.48(0.06-3.61)$ & 0.597 & & \\
\hline \multicolumn{5}{|l|}{ Vancomycin MIC } \\
\hline$\leq 1$ & Reference & & Reference & \\
\hline 2 & $0.21(0.05-0.90)$ & 0.054 & $0.19(0.04-1.00)$ & 0.050 \\
\hline Bacteremia & $0.28(0.07-1.10)$ & 0.076 & $0.31(0.07-1.40)$ & 0.128 \\
\hline
\end{tabular}

OR, odds ratio; $\mathrm{Cl}$, confidence interval; LD, loading dose; CRRT, continuous renal replacement therapy; CPR, cardiopulmonary resuscitation; ECMO, extracorporeal membrane oxygenation; MIC, minimum inhibitory concentration 
vancomycin $\mathrm{LD}$ was not (adjusted OR 0.91, 95\% CI: 0.27-2.99, $\mathrm{P}=0.870$ ).

Acute kidney injury tended to be higher in LD group compared to non-LD group ( $45.5 \%$ vs. $28.8 \%, \mathrm{P}=0.158$ ), while the difference was not statistically significant. In a multiple logistic regression model for variables associated with acute kidney injury, colistin use (adjusted OR 4.72, 95\% CI: $0.94-23.83, \mathrm{P}=0.060)$ and higher serum lactate (adjusted OR 1.39/mmol/L, 95\% CI: 0.95-2.05, $\mathrm{P}=0.095$ ) were marginally associated. However, vancomycin LD was not associated with increased risk of acute kidney injury.

\section{Subgroup analysis}

As LD group had a significantly higher baseline $\mathrm{CrCl}$, the possibility that a higher renal clearance negated a potential benefit of vancomycin LD was considered. Thus, a subgroup analysis was conducted after excluding patients with baseline $\mathrm{CrCl} \leq 30 \mathrm{~mL} / \mathrm{min}$. Baseline $\mathrm{CrCl}$ was similar between the two groups after matching by $\mathrm{CrCl}$. Weightbased dose was still significantly higher in LD group [24.5 (22.3-25.8) vs. 14.9 (12.7-18.7) mg/kg ABW, $\mathrm{P}<0.001$; Table S1]. However, time to PK target attainment and clinical outcome measures, including acute kidney injury, were not different between the two groups (Tables S2 and S3).

\section{Discussion}

The objective of this study was to evaluate an association of initial LD of vancomycin with improved clinical outcome in critically ill patients with microbiologically confirmed MRSA pneumonia. Although there was a higher initial dose of vancomycin administrated in LD group compared to non-LD group, there was neither significant difference in time to PK target attainment nor clinical cure of MRSA pneumonia between the two groups.

The current recommendation of initial vancomycin $\mathrm{LD}$ is based on PK studies and expert opinions $(6,9)$, but highquality data to guide the use of vancomycin $\mathrm{LD}$ are lacking (14). Previous PK studies reported that vancomycin LD has significant advantage in rapid attainment of PK without increased risk of adverse events $(11,12,23)$. However, to the authors' best knowledge, there is no comparative study evaluating the clinical benefit of vancomycin LD for MRSA pneumonia in critically ill patients to a control group. A recent retrospective cohort study by Jeon $e t$ al. showed that an initial daily dose of vancomycin $(<40 \mathrm{mg} / \mathrm{kg})$ was independently associated with the non-responsiveness of
MRSA pneumonia in hospitalized patients (24). However, the study evaluated daily doses of vancomycin on the first day in patients with MRSA pneumonia and compared them by clinical responsiveness. In addition, the majority of patients received the initial daily dose of vancomycin $(<40 \mathrm{mg} / \mathrm{kg})$ and the mean daily dose was not different between responders and non-responders. In the present study, the clinical outcomes in patients who received vancomycin $\mathrm{LD}$ were compared to those who did not, and vancomycin $\mathrm{LD}$ did not lead to significant improvements in clinical outcomes. There are several potential explanations for the observations in this study. First, the initial LD of vancomycin did not lead to more rapid attainment of target $\mathrm{C}_{\text {trough }}$, which has been widely regarded as a surrogate indicator of optimal AUC/MIC. This is likely to be the most important mechanism behind the observed similar clinical outcomes between LD and non-LD groups, as PK target attainment might be an intervening variable on the causal pathway from vancomycin LD to clinical outcome of MRSA pneumonia. Second, the proportion of subjects who reached PK target within $24 \mathrm{~h}$ in non-LD group $(30.9 \%)$ was higher than those reported in previous studies $(12,14,25)$. Interestingly, most patients $(88.9 \%)$ in this study had their first $\mathrm{C}_{\text {trough }}$ checked before the second or third dose of vancomycin, as the frequency of $\mathrm{C}_{\text {trough }}$ was left to the clinical pharmacist's discretion during the ICU multidisciplinary team rounding. More frequent tests than commonly recommended (before the fourth dose) might have helped patients in non-LD group reach PK target faster than would have reached otherwise $(9,26)$. In addition, baseline renal function was significantly better in LD group, so there is a possibility that higher renal clearance of vancomycin might have negated the benefit of a higher initial dose and resulted in a similar serum concentration. However, in a subgroup analysis of subjects with $\mathrm{CrCl}>30 \mathrm{~mL} / \mathrm{min}$ matched by $\mathrm{CrCl}$, there was no significant difference in time to PK target attainment and clinical outcomes between the two groups. Thus, it was unlikely that the difference in baseline renal function exerted a significant effect in the results of this study.

Furthermore, rapid PK target attainment was also not associated with clinical outcome in this study. Time to target attainment and the proportion of patients who reached PK target within $48 \mathrm{~h}$ was not significantly different between patients with or without clinical cure (Table 5). The clinical outcome of critically ill patients is determined by numerous factors, including comorbidities, severity of infection and organ failure, non-antibiotic treatment, and 
complications. Any single pharmacological intervention is unlikely to improve outcome dramatically in these severely ill patients, which has been demonstrated repeatedly (27). In addition, most clinical studies on vancomycin LD and clinical outcome were conducted in patients with bacteremia or septic shock, not with pneumonia $(14,15)$. Therefore, the added benefit of even faster target attainment might be too small to be detected, although the PK target was attained earlier in the subjects of this study compared to those in previous studies. In addition, previous studies have demonstrated that $\mathrm{C}_{\text {trough }}$ may not be an optimal surrogate for AUC values $(28,29)$. Therefore, the recent revised guideline on therapeutic monitoring of vancomycin no longer recommend through-only monitoring (10).

Lower vancomycin MIC of MRSA was the only independent predictive factor for clinical cure in this study, which is in concordance with previous studies $(30,31)$. It was suggested that vancomycin LD might be more helpful in cases with higher vancomycin MIC. However, a subgroup analysis could not be performed, as only nine patients were infected with MRSA isolates with MIC of $2 \mathrm{mg} / \mathrm{L}$. Future studies on infections caused by isolates with MIC $>1.5 \mathrm{mg} / \mathrm{L}$ may clarify the effect of vancomycin LD in this situation.

Many clinicians have a traditional fear of nephrotoxicity to give vancomycin LD (32). Critically ill patients are of particular concern, as they often harbor multiple risk factors for kidney injury. Also, in this study, patients in non-LD group had significantly worse baseline renal function, so it could be assumed that physicians avoided vancomycin LD in patients with impaired renal function due to concern for nephrotoxicity. However, no association was observed between vancomycin LD and risk for acute kidney injury. Patients in LD group showed nominally higher incidence of acute kidney injury, but the difference did not reach statistical significance. Vancomycin LD was not associated with acute kidney injury in multivariable analysis.

Although this study provided additional information on the clinical benefit of vancomycin LD in critically ill patients with MRSA pneumonia, there are several limitations that should be acknowledged. First, the sample size was relatively small. Thus, the results are underpowered to rule out the possibility of small difference in clinical outcome measures by vancomycin LD. Second, this was a retrospective study and the decision to use LD was at the discretion of treating physicians. Despite institutional protocol for vancomycin $\mathrm{LD}$, uptake of this recommendation had been poor at the authors' institution possibly due to clinicians' unfamiliarity with weight-based
LD. The authors tried to reduce the effect of potential bias and the difference in baseline characteristics by adjusted multivariate analysis. However, the potential for bias due to an unmeasured confounder remains. Third, although the most accurate PK/PD indicator for effective bacterial killing by vancomycin is known to be an AUC/MIC (8,33-35), AUC/MIC was not be measured. However, multiple measurement of peak and trough concentration is required to estimate AUC/MIC, making it less practical in realworld settings. $\mathrm{C}_{\text {trough }}$ was the only $\mathrm{PK}$ parameter that could be obtained, and it cannot be ascertained that $\mathrm{C}_{\text {trough }}$ reflected AUC closely in the population of this study. Last, some cases of polymicrobial infection have been included in our study, and it suggests the possibility that antibiotics other than vancomycin may affect clinical outcomes. However, the proportion of polymicrobial infection and microbiological cure rate were comparable between the two groups.

\section{Conclusions}

In conclusion, an initial LD of vancomycin was not associated with higher chance of clinical cure or more rapid attainment of PK target in critically ill patients with MRSA pneumonia. Further studies with prospective design are warranted to provide clearer evidence on the widely adopted practice of vancomycin LD in this population.

\section{Acknowledgments}

The authors thank Prof. Danbee Kang at Department of Clinical Research Design \& Evaluation, Samsung Advanced Institute for Health Sciences \& Technology (SAIHST), Sungkyunkwan University for comments regarding statistical analysis.

Funding: This study was supported by a grant from Samsung Medical Center grant (OTA1602901).

\section{Footnote}

Reporting Checklist: The authors have completed the STROBE reporting checklist. Available at http://dx.doi. org/10.21037/jtd-20-2243

Data Sharing Statement: Available at http://dx.doi. org/10.21037/jtd-20-2243

Conflicts of Interest: All authors have completed the ICMJE 
disclosure form (available at http://dx.doi.org/10.21037/ jtd-20-2243). The authors have no conflicts of interest to declare.

Ethical Statement: The authors are accountable for all aspects of the work in ensuring that questions related to the accuracy or integrity of any part of the work are appropriately investigated and resolved. The study was conducted in accordance with the Declaration of Helsinki (as revised in 2013). This study was approved by the institutional review board of Samsung Medical Center (IRB No. 2018-05-184). The requirement for informed consent was waived because of the observational nature of the study.

Open Access Statement: This is an Open Access article distributed in accordance with the Creative Commons Attribution-NonCommercial-NoDerivs 4.0 International License (CC BY-NC-ND 4.0), which permits the noncommercial replication and distribution of the article with the strict proviso that no changes or edits are made and the original work is properly cited (including links to both the formal publication through the relevant DOI and the license). See: https://creativecommons.org/licenses/by-nc-nd/4.0/.

\section{References}

1. Rubinstein E, Kollef MH, Nathwani D. Pneumonia caused by methicillin-resistant Staphylococcus aureus. Clin Infect Dis 2008;46 Suppl 5:S378-85.

2. Chiang CH, Pan SC, Yang TS, et al. Healthcare-associated infections in intensive care units in Taiwan, South Korea, and Japan: recent trends based on national surveillance reports. Antimicrob Resist Infect Control 2018;7:129.

3. Weiner-Lastinger LM, Abner S, Edwards JR, et al. Antimicrobial-resistant pathogens associated with adult healthcare-associated infections: Summary of data reported to the National Healthcare Safety Network, 2015-2017. Infect Control Hosp Epidemiol 2020;41:1-18.

4. Shorr AF, Tabak YP, Gupta V, et al. Morbidity and cost burden of methicillin-resistant Staphylococcus aureus in early onset ventilator-associated pneumonia. Crit Care 2006;10:R97.

5. Athanassa Z, Siempos, II, Falagas ME. Impact of methicillin resistance on mortality in Staphylococcus aureus VAP: a systematic review. Eur Respir J 2008;31:625-32.

6. Alvarez R, Lopez Cortes LE, Molina J, et al. Optimizing the Clinical Use of Vancomycin. Antimicrob Agents Chemother 2016;60:2601-9.
7. Gawronski KM, Goff DA, Brown J, et al. A stewardship program's retrospective evaluation of vancomycin AUC24/ MIC and time to microbiological clearance in patients with methicillin-resistant Staphylococcus aureus bacteremia and osteomyelitis. Clin Ther 2013;35:772-9.

8. Holmes NE, Turnidge JD, Munckhof WJ, et al. Vancomycin AUC/MIC ratio and 30-day mortality in patients with Staphylococcus aureus bacteremia. Antimicrob Agents Chemother 2013;57:1654-63.

9. Rybak MJ, Lomaestro BM, Rotschafer JC, et al. Vancomycin therapeutic guidelines: a summary of consensus recommendations from the infectious diseases Society of America, the American Society of HealthSystem Pharmacists, and the Society of Infectious Diseases Pharmacists. Clin Infect Dis 2009;49:325-7.

10. Rybak MJ, Le J, Lodise TP, et al. Therapeutic monitoring of vancomycin for serious methicillin-resistant Staphylococcus aureus infections: A revised consensus guideline and review by the American Society of HealthSystem Pharmacists, the Infectious Diseases Society of America, the Pediatric Infectious Diseases Society, and the Society of Infectious Diseases Pharmacists. Am J Health Syst Pharm 2020;77:835-64.

11. Alvarez O, Plaza-Plaza JC, Ramirez M, et al. Pharmacokinetic Assessment of Vancomycin Loading Dose in Critically Ill Patients. Antimicrob Agents Chemother 2017;61:e00280-17.

12. Truong J, Levkovich BJ, Padiglione AA. Simple approach to improving vancomycin dosing in intensive care: a standardised loading dose results in earlier therapeutic levels. Intern Med J 2012;42:23-9.

13. Zelenitsky S, Alkurdi N, Weber Z, et al. Preferential emergence of reduced vancomycin susceptibility in health care-associated methicillin-resistant Staphylococcus aureus isolates during continuous-infusion vancomycin therapy in an in vitro dynamic model. Antimicrob Agents Chemother 2011;55:3627-30.

14. Reardon J, Lau TT, Ensom MH. Vancomycin loading doses: a systematic review. Ann Pharmacother 2015;49:557-65.

15. Ortwine JK, Zasowski EJ, Pogue JM, et al. Relationship Status between Vancomycin Loading Dose and Treatment Failure in Patients with MRSA Bacteremia: It's Complicated. Infect Dis Ther 2019;8:627-40.

16. Flannery AH, Bachmeier H. Vancomycin-associated nephrotoxicity: unintentional consequences of a loading dose? Crit Care Med 2015;43:e154.

17. Roberts JA, Lipman J. Pharmacokinetic issues for 
antibiotics in the critically ill patient. Crit Care Med 2009;37:840-51; quiz 59.

18. Kalil AC, Metersky ML, Klompas M, et al. Management of Adults With Hospital-acquired and Ventilator-associated Pneumonia: 2016 Clinical Practice Guidelines by the Infectious Diseases Society of America and the American Thoracic Society. Clin Infect Dis 2016;63:e61-e111.

19. Levey AS, Bosch JP, Lewis JB, et al. A more accurate method to estimate glomerular filtration rate from serum creatinine: a new prediction equation. Modification of Diet in Renal Disease Study Group. Ann Intern Med 1999;130:461-70.

20. Joannidis M, Metnitz B, Bauer P, et al. Acute kidney injury in critically ill patients classified by AKIN versus RIFLE using the SAPS 3 database. Intensive Care Med 2009;35:1692-702.

21. Lopes JA, Jorge S. The RIFLE and AKIN classifications for acute kidney injury: a critical and comprehensive review. Clin Kidney J 2013;6:8-14.

22. Mickey RM, Greenland S. The impact of confounder selection criteria on effect estimation. Am J Epidemiol 1989;129:125-37.

23. Golenia BS, Levine AR, Moawad IM, et al. Evaluation of a vancomycin dosing nomogram based on the Modification of Diet in Renal Disease equation in intensive care unit patients. J Crit Care 2013;28:710-6.

24. Jeon YL, Kim MH, Yang HS, et al. Optimum initial loading dose of vancomycin for pneumonia caused by methicillin-resistant Staphylococcus aureus. J Infect 2016;72:115-8.

25. Rosini JM, Laughner J, Levine BJ, et al. A randomized trial of loading vancomycin in the emergency department. Ann Pharmacother 2015;49:6-13.

26. Liu C, Bayer A, Cosgrove SE, et al. Clinical practice guidelines by the infectious diseases society of america for the treatment of methicillin-resistant Staphylococcus aureus infections in adults and children. Clin Infect Dis

Cite this article as: Yoon JG, Huh K, Sohn YM, Park HJ, $\mathrm{Na}$ SJ, Jeon K. Effect of vancomycin loading dose on clinical outcome in critically ill patients with methicillin-resistant Staphylococcus aureus pneumonia. J Thorac Dis 2021;13(2):768777. doi: $10.21037 /$ jtd-20-2243 2011;52:e18-55.

27. Santacruz CA, Pereira AJ, Celis E, et al. Which Multicenter Randomized Controlled Trials in Critical Care Medicine Have Shown Reduced Mortality? A Systematic Review. Crit Care Med 2019;47:1680-91.

28. Mohr JF, Murray BE. Point: Vancomycin is not obsolete for the treatment of infection caused by methicillinresistant Staphylococcus aureus. Clin Infect Dis 2007;44:1536-42.

29. Pai MP, Neely M, Rodvold KA, et al. Innovative approaches to optimizing the delivery of vancomycin in individual patients. Adv Drug Deliv Rev 2014;77:50-7.

30. Sakoulas G, Moise-Broder PA, Schentag J, et al. Relationship of MIC and bactericidal activity to efficacy of vancomycin for treatment of methicillin-resistant Staphylococcus aureus bacteremia. J Clin Microbiol 2004;42:2398-402.

31. Soriano A, Marco F, Martinez JA, et al. Influence of vancomycin minimum inhibitory concentration on the treatment of methicillin-resistant Staphylococcus aureus bacteremia. Clin Infect Dis 2008;46:193-200.

32. Filippone EJ, Kraft WK, Farber JL. The Nephrotoxicity of Vancomycin. Clin Pharmacol Ther 2017;102:459-69.

33. Jung Y, Song KH, Cho Je, et al. Area under the concentration-time curve to minimum inhibitory concentration ratio as a predictor of vancomycin treatment outcome in methicillin-resistant Staphylococcus aureus bacteraemia. Int J Antimicrob Agents 2014;43:179-83.

34. Kullar R, Davis SL, Levine DP, et al. Impact of Vancomycin Exposure on Outcomes in Patients With Methicillin-Resistant Staphylococcus aureus Bacteremia: Support for Consensus Guidelines Suggested Targets. Clin Infect Dis 2011;52:975-81.

35. Lodise TP, Drusano GL, Zasowski E, et al. Vancomycin Exposure in Patients With Methicillin-Resistant Staphylococcus aureus Bloodstream Infections: How Much Is Enough? Clin Infect Dis 2014;59:666-75. 


\section{Supplementary}

Table S1 Vancomycin dose and $\mathrm{C}_{\text {trough }}$ measurement between vancomycin loading dose (LD) and non-LD groups in subgroup analysis with patients who had creatinine clearance $(\mathrm{CrCl})$ of $>30 \mathrm{~mL} / \mathrm{min}$ and were matched by $\mathrm{CrCl}$

\begin{tabular}{|c|c|c|c|}
\hline Characteristics & LD group $(n=20)$ & Non-LD group $(n=20)$ & $P$ value \\
\hline \multicolumn{4}{|l|}{ First dose of vancomycin } \\
\hline Median dose per weight, $\mathrm{mg} / \mathrm{kg}$ & $24.5(22.3-25.8)$ & $14.9(12.7-18.7)$ & $<0.001$ \\
\hline \multicolumn{4}{|c|}{ Groups by median dose per weight, mg/kg } \\
\hline $15-20$ & $2(10.0)$ & $7(35.0)$ & \\
\hline $20-25$ & $12(60.0)$ & $3(15.0)$ & \\
\hline $25-30$ & $5(25.0)$ & $0(0.0)$ & \\
\hline$>30$ & $1(5.0)$ & $0(0.0)$ & \\
\hline
\end{tabular}

Values are median with interquartile range or $\mathrm{n}(\%)$.

Table S2 Proportion of pharmacokinetic target (trough concentration $\geq 15 \mathrm{mg} / \mathrm{L}$ ) attainment between vancomycin loading dose (LD) and nonLD groups in subgroup analysis with patients who had creatinine clearance $(\mathrm{CrCl})$ of $>30 \mathrm{~mL} / \mathrm{min}$ and were matched by $\mathrm{CrCl}$

\begin{tabular}{|c|c|c|c|}
\hline Characteristics & LD group $(n=20)$ & Non-LD group $(n=20)$ & $P$ value \\
\hline Target attainment within $24 \mathrm{~h}$ & $6(31.6)$ & $8(44.4)$ & 0.420 \\
\hline Target attainment within $48 \mathrm{~h}$ & $10(52.6)$ & $10(55.6)$ & 0.858 \\
\hline
\end{tabular}

Values are median with interquartile range or $\mathrm{n}(\%)$. OR, odds ratio; $\mathrm{Cl}$, confidence interval.

Table S3 Clinical outcomes between vancomycin loading dose (LD) and non-LD groups in subgroup analysis with patients who had creatinine clearance $(\mathrm{CrCl})$ of $>30 \mathrm{~mL} / \mathrm{min}$ and were matched by $\mathrm{CrCl}$

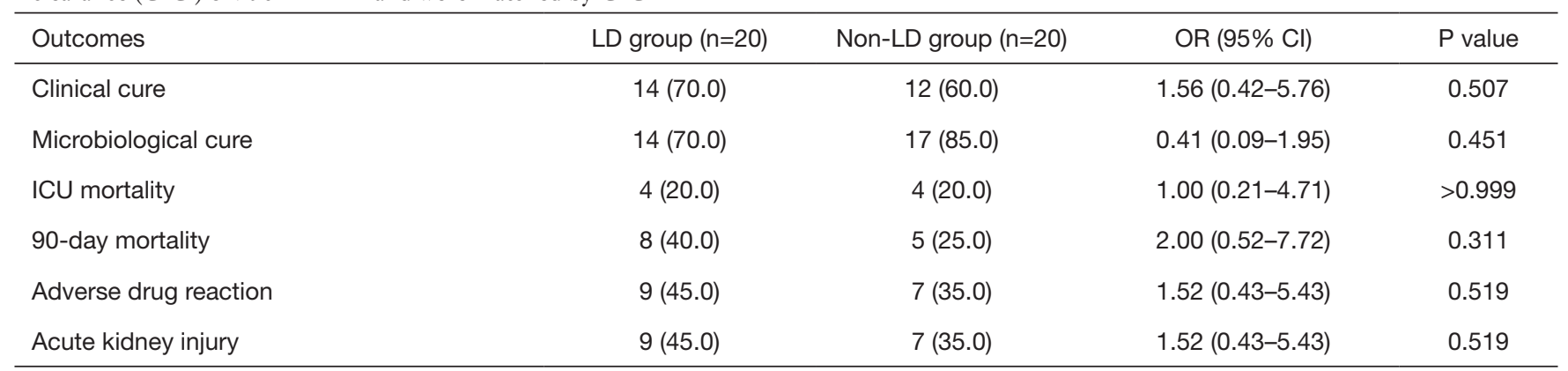

OR, odds ratio; $\mathrm{Cl}$, confidence interval; ICU, intensive care unit. 